\title{
Clinical Outcomes after Reverse Total Shoulder Arthroplasty According to Primary Diagnosis
}

\author{
Jung Youn Kim, MD, Yong Girl Rhee, MD*,*, Sung-Min Rhee, MD* \\ Department of Orthopaedic Surgery, Kangnam Sacred Heart Hospital, Hallym University College of Medicine, Seoul, \\ * Shoulder and Elbow Clinic, Department of Orthopaedic Surgery, Kyung Hee University College of Medicine, Seoul, Korea
}

Background: To compare the clinical outcomes of reverse total shoulder arthroplasty (RTSA) according to the primary diagnosis. Methods: In 98 shoulders (97 patients), RTSA was performed for cuff tear arthropathy (45), massive rotator cuff tear without glenohumeral arthritis (31), posttraumatic arthritis (9), primary osteoarthritis (6), rheumatoid arthritis (4), and arthritis due to infection sequelae (3). The average age of the patients at surgery was 68.9 years (range, 46-84 years). The mean follow-up duration was 48.4 months (range, 24-85 months).

Results: In the overall series, the mean subjective pain score (visual analog scale) during motion decreased from 5.2 preoperatively to 1.8 at 2 years of follow-up. There were significant improvements in active forward flexion (preoperatively $51.5^{\circ}$ to $121.8^{\circ}$ at 2 years of follow-up). The average Constant score improved from 35.4 points to 57.8 points and UCLA score improved from 13.4 points to 28.8 points. The Constant score and UCLA score were 60.8 and 31.0 points, respectively, in patients with rheumatoid arthritis. The Constant score and UCLA score were 58.4 and 29.1 points, respectively, in patients with cuff tear disease and 55.7 and 27.7 points, respectively, in patients with posttraumatic arthritis. Patients' subjective satisfaction was 86.8 points in the overall series; highest in the patients with arthritis by infection sequelae (96.7 points) and lowest in the patients with posttraumatic arthritis (82.2 points). In terms of complications, there were 17 cases (17.3\%) of scapular notching and 2 patients with suprascapular nerve irritation symptom, but no patients with permanent neuropathy.

Conclusions: The range of forward flexion and abduction motion, pain relief, and functionality were improved after RTSA in not only patients with cuff tear disease but also those with other arthritic diseases. There was no difference in the clinical outcomes of RTSA between patients with cuff tear disease and those with other arthritic diseases.

Keywords: Shoulder, Rotator cuff tears, Rotator cuff tear arthropathy, Shoulder arthroplasty replacement

Reverse total shoulder arthroplasty (RTSA) was originally developed to address the complex problems of cuff tear arthropathy. ${ }^{1)}$ Many studies have reported successful results after RTSA for cuff tear arthropathy. ${ }^{2,3)}$ Al-Hadithy

Received November 20, 2019; Accepted April 12, 2020

Correspondence to: Sung-Min Rhee, MD

Department of Orthopaedic Surgery, Kyung Hee University College of Medicine, 23 Kyungheedae-ro, Dongdaemun-gu, Seoul 02447, Korea

Tel: +82-2-958-8370, Fax: +82-2-964-3865

E-mail: starsmstar@khmc.or.kr

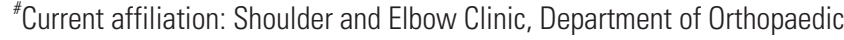
Surgery, Myongji Hospital, Goyang, Korea et al. $^{2)}$ reported that the mean Constant score improved from 34.2 to 71.0 points with a mean follow-up of 5 years. Moreover, Favard et al. ${ }^{3)}$ described a 10 -year survival rate of $89 \%$ after RTSA in 484 shoulders with cuff tear arthropathy in a multicenter study that included revision surgery.

On the basis of these excellent outcomes of RTSA in patients with cuff tear arthropathy, the indications for this treatment method have been widened as implants are improved and surgeons gain more experience, and RTSA has been used for treatment and revision of other diseases and fractures. ${ }^{4)}$ Recently, the indications for RTSA have been expanded to include young, higher demand patients with shoulder problems. ${ }^{5}$ Holcomb et al. ${ }^{6}$ described the 
Kim et al. Reverse Total Shoulder Arthroplasty According to Primary Diagnosis

Clinics in Orthopedic Surgery • Vol. 12, No. 4, $2020 \bullet$ www.ecios.org

successful use of RTSA in the treatment of rheumatoid arthritis. However, Boileau et al. ${ }^{7)}$ reported poorer clinical outcomes and higher complication and revision rates than those reported for cuff tear arthropathy when RTSA was performed in patients with posttraumatic arthritis or failure of a revision arthroplasty, and Wall et al. ${ }^{8)}$ reported that patients with posttraumatic arthritis and those undergoing revision arthroplasty had less improvement and higher complication rates than patients with other etiologies when RTSA outcomes were compared across various etiologies including primary osteoarthritis, posttraumatic arthritis, tumor, acute fracture, and rheumatoid arthritis. Kilic et al. ${ }^{9)}$ compared the use of standard total shoulder arthroplasty and RTSA for posttraumatic arthritis and showed that RTSA led to an improvement in postoperative results.

Although RTSA has been attempted for the treatment of various shoulder problems, most of the reports still involved patients with cuff tear arthropathy. Moreover, long-term follow-up data are lacking, and there are only a few reports for other conditions. In particular, few studies have compared the outcomes of patients who have undergone RTSA for arthritis due to primary cuff tear disease with those of patients with arthritis due to other lesions. ${ }^{8)}$ For this reason, in contrast with patients with cuff tear arthropathy or a massive rotator cuff tear, it is difficult to determine the outcomes of RTSA for patients with other arthritic disease.

The purpose of this study was to compare the clinical outcomes after RTSA according to the primary diagnosis. We hypothesized that there would be little difference in the outcomes of RTSA between patients with cuff tear disease and those with other arthritic diseases and also that the clinical outcomes of RTSA for patients with posttraumatic arthritis or arthritis due to infection sequelae would be poorer than those for patients with cuff tear disease.

\section{METHODS}

This study is retrospective in nature and was approved by the Institutional Review Board of Kyung Hee University Hospital (IRB No. KMC IRB 1542-07). The requirement of informed consent was exempted.

\section{Patient Selection}

A series of RTSA procedures performed by a single senior surgeon (YGR) at Kyung Hee University Hospital from January 2008 to June 2013 were reviewed retrospectively. The prosthesis used was the Aequalis system (Tornier, Montbonnot-Saint-Martin, France) implanted through a deltopectoral approach. During this period, consecutive cases that were followed up for at least 24 months were analyzed. Four cases were excluded due to short followup duration. Finally, 98 prostheses were implanted in 97 patients, with 1 patient receiving bilateral implants. The average age of the patients at surgery was 68.9 years (range, 46-84 years). Sixty-seven procedures were performed in female patients and 31 were performed in male patients. Seventy-two prostheses were placed in the right shoulder and 26 were placed in the left shoulder. The dominant shoulder was involved in 77 cases, and the nondominant shoulder was involved in 21 cases.

There were 45 shoulders (44 patients) with cuff tear arthropathy and 31 shoulders with a massive rotator cuff tear without glenohumeral arthritis (Fig. 1). Moreover, there were 9 shoulders with posttraumatic arthritis (Fig. 2), 6 with primary osteoarthritis, 4 with rheumatoid arthritis (Fig. 3), and 3 with arthritis from infection sequelae with a massive rotator cuff tear. Patients with cuff tear arthropathy or a massive rotator cuff tear without glenohumeral arthritis were designated as the cuff tear disease group, whereas patients with other etiologies were categorized as the other arthritic disease group. Patients who were classified as having posttraumatic arthritis included those who had glenohumeral arthritis and a history of a fracture or dislocation of the humerus.

\section{Preoperative and Postoperative Evaluations}

All patients underwent preoperative evaluations at 1 day before the surgery. Postoperative evaluations were performed regularly on an outpatient basis, and the results of the 2-year follow-up were analyzed. The preoperative and postoperative subjective pain scores were measured using the visual analog scale (VAS), with 0 indicating no pain and 10 indicating extremely severe pain. For the shoulder range of motion (ROM), forward flexion, external rotation at the side, internal rotation to the back, and abduction were assessed before and after the operation. The Constant score ${ }^{10)}$ and the Shoulder Rating Scale of the University of California at Los Angeles (UCLA) ${ }^{11)}$ were used for clinical assessment. Muscle strength was quantified with a portable, handheld Nottingham Mecmesin Myometer (Mecmesin Co., Nottingham, UK). The elevation and abduction strength were tested with the patient in the seated position with the arm flexed to $90^{\circ}$ in the scapular plane. External and internal rotations were tested with the shoulder in the neutral position and the elbow in $90^{\circ}$ flexion. At the 2-year follow-up, the patients were asked to evaluate their subjective satisfaction with the treatment on a 100-point scale, with 100 indicating full satisfaction. 
Kim et al. Reverse Total Shoulder Arthroplasty According to Primary Diagnosis

Clinics in Orthopedic Surgery • Vol. 12, No. 4, $2020 \bullet$ www.ecios.org

Radiographs including anteroposterior views of the glenohumeral joint in neutral rotation, internal rotation, and external rotation, as well as axillary views were evaluated for all cases preoperatively, postoperatively, and at each follow-up visit. The radiographs were assessed for evidence of radiolucency and loosening. The presence of radiolucency was noted under the base plate and around the screw and peg. Loosening of the implant was considered to be present when either the base plate or the stem of the humerus was displaced. Scapular notching, which is a defect of the bone in the inferior part of the glenoid component, was classified according to Sirveaux's grading. ${ }^{12)}$

\section{Statistical Analysis}

Analysis of variance was used to compare the functional scores, ROMs, and muscle strength in the various etiologic groups. Independent $t$-test, chi-square test, and MannWhitney $U$-test were used to compare the clinical and radiological outcomes. Significance was set at a level of 0.05 with associated $95 \%$ confidence intervals. For multiple comparison statistical analysis, corrected $p$-values by Bonferroni correction were used. RTSA was done bilaterally in 1 patient, and each replacement procedure was considered a separate case. The IBM SPSS ver. 20.0 (IBM Corp., Armonk, NY, USA) was used for all statistical analyses.

\section{RESULTS}

\section{Overall Clinical Outcomes}

The mean subjective pain score (VAS) during motion decreased from 5.2 (range, $0-10$ ) preoperatively to 1.8 (range, $0-5)$ at the time of the 2-year follow-up $(p<0.001)$. There were significant improvements in active forward flexion (51.5 $5^{\circ}$ preoperatively to $121.8^{\circ}$ at the 2 -year follow-up). However, there were no differences in external rotation at the side $\left(37.9^{\circ}\right.$ preoperatively to $35.5^{\circ}$ at the 2 -year followup) and internal rotation motion (L2.8 preoperatively to L3.4 at the 2-year follow-up). The average Constant score improved from 35.4 points (range, $14-56$ points) before surgery to 57.8 points (range, $38-83$ points) at the 2 -year follow-up. The average UCLA score improved from 13.4 points (range, 4-22 points) preoperatively to 28.8 points (range, 20-35 points) at the 2-year follow-up. Muscle strength improved significantly after RTSA in forward flexion, external rotation, internal rotation, and abduction. The patients' mean subjective satisfaction score was 86.8 points (range, $60-100$ points), and 84 shoulders (85.7\%) had a satisfaction score of 80 points (Table 1).

Table 1. Preoperative and Postoperative Clinical Outcomes

\begin{tabular}{|c|c|c|c|}
\hline Variable & Preoperative & 2-Year follow-up & $p$-value \\
\hline UCLA score & $13.4 \pm 3.9$ & $28.8 \pm 3.8$ & $<0.001^{*}$ \\
\hline \multicolumn{4}{|l|}{$\mathrm{ROM}\left({ }^{\circ}\right)$} \\
\hline ER at the side & $37.9 \pm 10.5$ & $35.5 \pm 10.5$ & 0.116 \\
\hline IRp & $\mathrm{L} 2.8 \pm 2.9$ & $\mathrm{~L} 3.4 \pm 1.3$ & 0.062 \\
\hline Abduction & $96.9 \pm 18.1$ & $108.6 \pm 16.6$ & $<0.001^{*}$ \\
\hline $\mathbb{I R}$ & $4.0 \pm 2.0$ & $6.1 \pm 2.7$ & $<0.001^{*}$ \\
\hline Abduction & $2.2 \pm 1.4$ & $5.0 \pm 3.1$ & $<0.001^{*}$ \\
\hline
\end{tabular}

Values are presented as mean \pm standard deviation.

VAS: visual analog scale, UCLA: University of California at Los Angeles, ROM: range of motion, FF: forward flexion, ER: external rotation, IRp: internal rotation to the back, IR: internal rotation.

*Statistically significant. 
Kim et al. Reverse Total Shoulder Arthroplasty According to Primary Diagnosis

Clinics in Orthopedic Surgery • Vol. 12, No. 4, $2020 \bullet$ www.ecios.org

\section{Comparison of Results between Patients with Cuff Tear Arthropathy and Those with Massive Rotator Cuff Tear Only}

The mean VAS score during motion was 1.6 (range, 0-4) in patients with cuff tear arthropathy and 2.0 (range, 0-5) in patients with massive rotator cuff tear only at the 2-year follow-up. There was no significant difference in pain at the 2-year follow-up $(p=0.596)$. Concerning the mean $\mathrm{ROM}$ at the 2-year follow-up, there were no differences in active forward flexion, external rotation at the side, internal rotation, and abduction. Moreover, there were no differences in the average Constant score and the average UCLA score between patients with cuff tear arthropathy and those with massive rotator cuff tear only at the 2-year follow-up. Muscle strength and the patients' subjective satisfaction showed no difference in patients with cuff tear arthropathy and those with massive rotator cuff tear only at the 2-year follow-up.

\section{Clinical Outcomes According to the Primary Diagnosis}

Table 2 shows the clinical outcomes according to the primary diagnosis at the 2-year follow-up. At the 2-year follow-up, patients who underwent RTSA for arthritis due to infection sequelae and rheumatoid arthritis showed the best outcomes for pain relief, whereas patients who underwent RTSA for primary osteoarthritis and posttraumatic arthritis showed inferior outcomes for pain relief. Patients who underwent RTSA for rheumatoid arthritis showed more favorable functional outcomes than did patients with cuff tear disease, whereas patients with primary osteoarthritis, posttraumatic arthritis, and arthritis due to infection sequelae showed outcomes inferior to those of patients with cuff tear disease. However, these results did not reach statistical significance. Subjective satisfaction was the highest for patients who underwent RTSA for arthritis due to infection sequelae (96.7 points) and was the lowest for patients who underwent RTSA for posttraumatic arthritis (82.2 points).

Based on the above results, patients who underwent RTSA for rheumatoid arthritis showed superior clinical outcomes in pain relief, functionality, and patient satisfaction than patients with cuff tear disease, whereas patients with posttraumatic arthritis or primary osteoarthritis showed inferior clinical outcomes. However, statistical significance was not achieved owing to the small sample size.

\section{Comparison of Results between Patients with Cuff Tear Disease and Those with Other Arthritic Diseases}

The mean VAS during motion was 1.8 (range, $0-5$ ) in patients with cuff tear disease and 1.9 (range, $0-5$ ) in those with other arthritic diseases at the 2-year follow-up. There was no significant difference in pain score at the 2-year follow-up $(p=1.000)$. There were no differences in the average Constant score (58.4 points vs. 52.0 points, $p=0.702$ ) and UCLA score (29.1 points vs. 27.0 points, $p=0.540$ ). Concerning the mean ROM at the 2-year follow-up, there were no differences in active forward flexion $\left(121.8^{\circ} \mathrm{vs}\right.$. $\left.115.5^{\circ}\right)$, external rotation at the side $\left(35.5^{\circ}\right.$ vs. $\left.35.5^{\circ}\right)$, internal rotation to the back (L3.6 vs. L3.6), and abduction $\left(108.8^{\circ}\right.$ vs. $\left.108.2^{\circ}\right)$. The muscle strength and patients subjective satisfaction (87.0 points vs. 86.4 points) showed no difference between patients with cuff tear disease and those with other arthritic diseases (Table 3).

\section{Complications}

In the overall series, there were 17 cases $(17.3 \%)$ of scapu-

Table 2. Clinical Outcomes According to Primary Diagnosis at the 2-Year Follow-up

\begin{tabular}{|c|c|c|c|c|c|}
\hline Variable & No. & VAS & Constant & UCLA & Satisfaction \\
\hline CTA & 45 & $1.6 \pm 1.4$ & $57.4 \pm 7.7$ & $29.2 \pm 3.5$ & $88.2 \pm 10.3$ \\
\hline Massive RCT & 31 & $2.0 \pm 1.6$ & $59.9 \pm 9.5$ & $29.0 \pm 4.0$ & $85.2 \pm 11.8$ \\
\hline PTA & 9 & $2.4 \pm 1.9$ & $55.7 \pm 10.9$ & $27.7 \pm 4.9$ & $82.2 \pm 13.9$ \\
\hline Primary $\mathrm{OA}$ & 6 & $2.5 \pm 1.0$ & $52.3 \pm 9.4$ & $26.5 \pm 3.7$ & $85.0 \pm 5.5$ \\
\hline RA & 4 & $0.8 \pm 1.0$ & $60.8 \pm 3.1$ & $31.0 \pm 2.0$ & $90.0 \pm 14.1$ \\
\hline Infection & 3 & $0.7 \pm 1.2$ & $53.7 \pm 9.2$ & $27.7 \pm 4.9$ & $96.7 \pm 5.8$ \\
\hline Overall & 98 & $1.8 \pm 1.5$ & $57.8 \pm 8.8$ & $28.8 \pm 3.8$ & $86.8 \pm 11.1$ \\
\hline
\end{tabular}

Values are presented as mean \pm standard deviation.

VAS: visual analog scale, Constant: Constant score, UCLA: University of California at Los Angeles, Satisfaction: patients' subjective satisfaction, CTA: cuff tear arthropathy, Massive RCT: irreparable massive rotator cuff tear without glenohumeral arthritis, PTA: posttraumatic arthritis, 0A: osteoarthritis, RA: rheumatoid arthritis, Infection: arthritis due to infection sequelae. 
Kim et al. Reverse Total Shoulder Arthroplasty According to Primary Diagnosis

Clinics in Orthopedic Surgery • Vol. 12, No. 4, $2020 \bullet$ www.ecios.org

Table 3. Comparison between Cuff Tear Disease and Other Arthritic Diseases at the 2-Year Follow-up

\begin{tabular}{|c|c|c|c|}
\hline Variable & Cuff tear disease & Other arthritic diseases & $p$-value* \\
\hline No. of cases & 76 & 22 & \\
\hline VAS & $1.8 \pm 1.5$ & $1.9 \pm 1.6$ & 1.000 \\
\hline Constant score & $58.4 \pm 9.3$ & $52.0 \pm 10.2$ & 0.702 \\
\hline UCLA score & $29.1 \pm 3.9$ & $27.0 \pm 4.9$ & 0.540 \\
\hline \multicolumn{4}{|l|}{$\operatorname{ROM}\left(^{\circ}\right)$} \\
\hline Active FF & $121.8 \pm 28.9$ & $115.5 \pm 31.8$ & 1.000 \\
\hline ER at the side & $35.5 \pm 10.5$ & $35.5 \pm 9.5$ & 1.000 \\
\hline IRp & L3.6 \pm 1.3 & $\mathrm{~L} 3.6 \pm 1.6$ & 1.000 \\
\hline Abduction & $108.8 \pm 17.6$ & $108.2 \pm 13.0$ & 1.000 \\
\hline \multicolumn{4}{|c|}{ Muscle strength (lb) } \\
\hline Active FF & $5.7 \pm 2.9$ & $4.9 \pm 2.4$ & 0.612 \\
\hline ER at the side & $5.9 \pm 2.9$ & $4.9 \pm 2.1$ & 0.422 \\
\hline IRp & $6.2 \pm 2.9$ & $5.7 \pm 2.5$ & 1.000 \\
\hline Abduction & $5.2 \pm 3.2$ & $4.3 \pm 2.5$ & 0.514 \\
\hline Satisfaction & $87.0 \pm 11.0$ & $86.4 \pm 11.8$ & 1.000 \\
\hline
\end{tabular}

Values are presented as mean \pm standard deviation.

VAS: visual analog scale, UCLA: University of California at Los Angeles, ROM: range of motion, FF: forward flexion, ER: external rotation, IRp: internal rotation to the back.

${ }^{*}$ Adjusted $p$-value of Bonferroni correction for multiple comparisons.
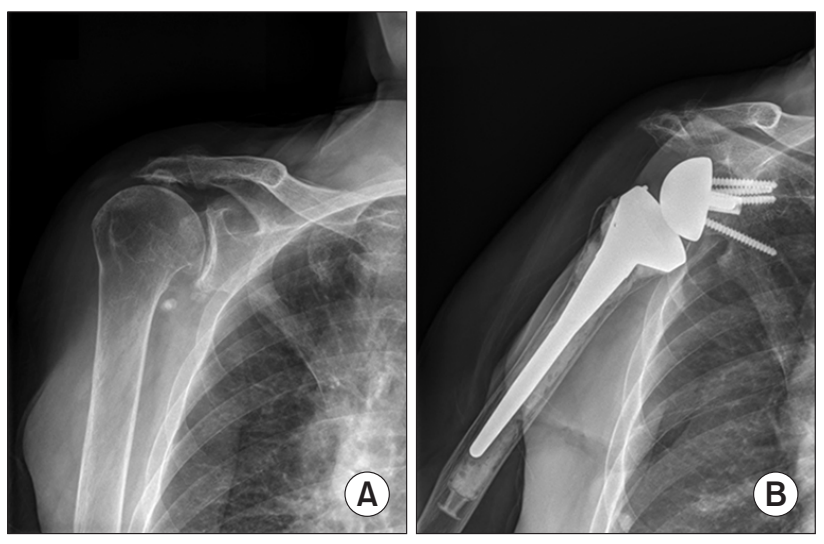

Fig. 1. Radiographs of a patient with cuff tear arthropathy. (A) Preoperative true anteroposterior view. (B) Two-year follow-up true anteroposterior view.

lar notching. According to Sirveaux's grading, ${ }^{12)}$ there were 12 shoulders in grade 1,4 shoulders in grade 2 , and 1 shoulder in grade 3 . The 17 cases of scapular notching showed no difference in functional outcomes among the total patients, with an average UCLA score of 28.2 points and Constant score of 59.4 points at the 2-year follow-up.

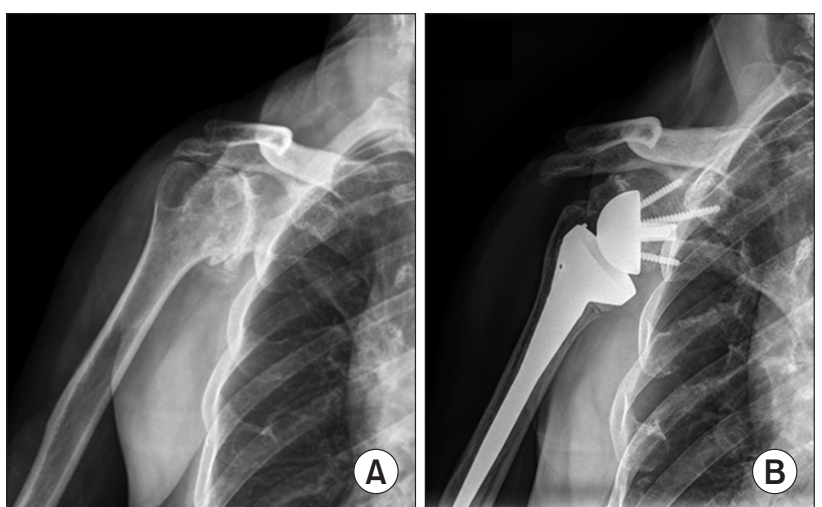

Fig. 2. Radiographs of a patient with posttraumatic arthritis. (A) Preoperative true anteroposterior view. (B) Two-year follow-up true anteroposterior view.

Although an acromion tip avulsion fracture occurred in 1 patient who underwent RTSA for cuff tear arthropathy, pain disappeared after 6 weeks of conservative treatment and ROM also returned to pre-fracture levels. A nontraumatic acromial spine fracture occurred in the 7th postoperative month in 1 patient who underwent RTSA for 

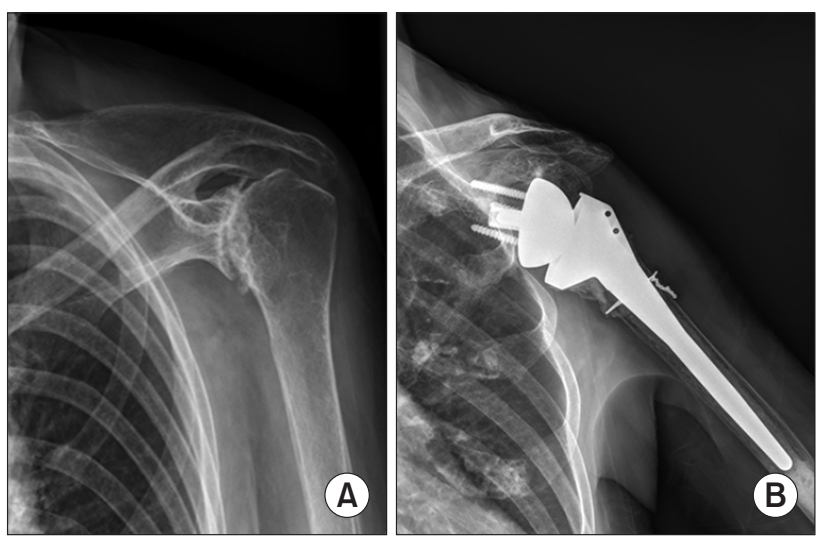

Fig. 3. Radiographs of a patient with rheumatoid arthritis. (A) Preoperative true anteroposterior view. (B) Two-year follow-up true anteroposterior view.

a massive rotator cuff tear; however, the patient showed improved functionality at the 2-year follow-up (postoperative 25 months) without additional surgery. There were 2 patients with suprascapular nerve irritation symptoms such as pain radiating to the neck and arms and aggravation due to shoulder movement. In 2 patients, infraspinatus muscle atrophy was observed in patients with cuff tear arthropathy; however, the symptom was relieved after a steroid injection at the spinoglenoid notch. There was no permanent nerve lesion. There was no dislocation in the early postoperative period; however, 1 anterior dislocation was observed in patients with cuff tear arthropathy after excessive external rotation motion at postoperative 2 years. Nevertheless, there was no need for additional surgery after the closed reduction.

\section{DISCUSSION}

Although various surgical treatments have been attempted for cuff tear arthropathy, including simple debridement, ${ }^{13)}$ tuberoplasty, ${ }^{14)}$ partial repair, ${ }^{15)}$ latissimus dorsi transfer, ${ }^{16)}$ and hemiarthroplasty or total shoulder arthroplasty, ${ }^{17)}$ these treatments have failed to achieve satisfactory functional outcomes and ROM, and thus RTSA is used as an alternative treatment method. ${ }^{18,19)}$ The indications for RTSA have recently been expanded beyond cuff tear arthropathy to include various shoulder problems such as posttraumatic arthritis, ${ }^{8}$ primary glenohumeral osteoarthritis, ${ }^{20)}$ rheumatoid arthritis, ${ }^{6,21)}$ arthritis from infection sequelae, ${ }^{22)}$ acute fracture, and revision arthroplasty, ${ }^{23)}$ and excellent outcomes have been reported. Moreover, RTSA could be a treatment option not only in patients with chronic locked anterior shoulder dislocation but also in patients with malignant humeral tumor. ${ }^{24,25)}$

Wall et al. ${ }^{8)}$ studied 186 patients who underwent RTSA for various etiologies with an average follow-up of 39.9 months: Constant scores improved from 23 points preoperatively to 60 points postoperatively and 173 patients (93\%) were satisfied or highly satisfied with the surgical outcome. In the current series, the patients showed improvements of 22.4 points and 15.4 points in the Constant and UCLA scores, respectively; a $70.3^{\circ}$ improvement in active forward flexion; and an $86 \%$ outcome satisfaction, as indicated by a score of $\geq 80$ points. These clinical outcomes are similar to or better than those in previous reports on RTSA carried out for the treatment of rotator cuff tear arthropathy. ${ }^{26)}$

Holcomb et al. ${ }^{6)}$ performed a prospective study for an average of 3 years for 21 shoulders that underwent RTSA for rheumatoid arthritis. In addition to improved functionality and increased ROM for active forward flexion from $52^{\circ}$ to $126^{\circ}$ and for abduction from $55^{\circ}$ to $116^{\circ}$, they also found an increase in ROM for external rotation $\left(19^{\circ}-33^{\circ}\right)$ and internal rotation to the back (S1-L4). Also, in our study, patients who underwent RTSA for rheumatoid arthritis showed more favorable functional outcomes and higher subjective satisfaction than did patients with cuff tear disease. Although the results did not achieve statistical significance owing to the small number of cases, based on our results and those of previous studies, RTSA appears to be a good treatment method for patients with rheumatoid arthritis accompanied by a massive rotator cuff tear.

Willis et al. ${ }^{27)}$ and Martinez et al. ${ }^{28)}$ described the use of RTSA in patients with malunion of the proximal humerus and posttraumatic arthritis. They reported that RTSA improved function and active forward flexion to $52^{\circ}$ in patients with posttraumatic arthritis. Our study also showed a Constant score increase of 24.5 points and an increase of $71^{\circ}$ in active forward flexion for posttraumatic arthritis patients. However, this represents a lesser improvement than the outcomes for patients with other etiologies. Our results demonstrated an average satisfaction of 82.2 points in posttraumatic arthritis patients, suggesting that RTSA is a surgical method that could be used even in patients with posttraumatic arthritis accompanied by a massive rotator cuff tear.

Frankle et al. ${ }^{26)}$ investigated the short-term 2-year follow-up outcomes in 60 patients with primary glenohumeral joint osteoarthritis with a rotator cuff tear and reported an improvement in functional outcomes, VAS, and ROM for forward flexion and abduction, as well as satisfactory outcomes in 57 patients (95\%), a complica- 
Kim et al. Reverse Total Shoulder Arthroplasty According to Primary Diagnosis

Clinics in Orthopedic Surgery • Vol. 12, No. 4, $2020 \bullet$ www.ecios.org

tion rate of $17 \%$, and a surgery failure rate of $12 \%$. Cuff et al. ${ }^{29)}$ described an increase in the functional score, forward flexion, abduction, and external rotation motion, as well as satisfaction in surgical outcomes for $94 \%$ of patients at a short-term follow-up of 2 years in 96 cases of primary osteoarthritis accompanied by a rotator cuff tear. Although the patients with primary osteoarthritis who were included in our study showed an increase in the functional score, forward flexion, abduction, and external rotation motion, their 2-year mean Constant and UCLA scores were the lowest among all etiologies. The Constant score in the primary osteoarthritis patients was $52.3 \pm 9.4$ at the 2-year follow-up. However, all primary osteoarthritis patients in this study showed a satisfaction score of $\geq 80$ points. Therefore, RTSA can be thought to be an effective surgical method for patients with primary osteoarthritis.

Cuff et al. ${ }^{30)}$ retrospectively reviewed 21 patients (22 shoulders) with arthritis due to deep infection and reported improvements of forward flexion $\left(43.1^{\circ}-79.5^{\circ}\right)$ motion at a mean follow-up of 43 months after debridement and RTSA. In our study, patients who underwent arthroplasty for arthritis due to infection sequelae showed alleviation of pain and improvement of forward flexion $\left(46.7^{\circ}-126.7^{\circ}\right)$, whereas the functional outcomes were similar to those of patients with cuff tear disease; these patients had the highest subjective satisfaction. These results show that RTSA could alleviate pain, increase ROM, and provide high satisfaction for patients with joint destruction due to septic arthritis or other infection sequelae.

Although this study involved 17 cases (17\%) of scapular notching, this condition was due to various underlying diseases, and thus it was not possible to find a correlation between the primary diagnosis and scapular notching. At the last follow-up in the 17 cases with scapular notching, there was no difference in functional outcome from the overall patient group, with an average Constant score of 59.4 points and an average UCLA score of 28.2 points, and these results were similar to those of a previous study reporting no particular association between scapular notching and pain or clinical outcomes. ${ }^{31)}$ But in this study, we were unable to find any association between the occurrence of complications and the primary diagnosis.

Our study has a few limitations. First, it has limitations inherent to studies of a retrospective nature. However, we conducted a retrospective analysis of data from prospectively collected patients. Second, the number of other arthritic diseases (posttraumatic arthritis, primary osteoarthritis, RA, and infection sequalae) was small. In addition, the minimum follow-up duration of 24 months is relatively short for investigating the results of arthroplasty. A longer-term follow-up study is necessary to provide an adequate assessment of the implant longevity in RTSA. Nevertheless, this study is valuable because there are few studies comparing the results of RSTA in arthritic diseases other than cuff tear disease.

In addition to patients with cuff tear disease, those with other arthritic diseases also showed significant improvements in the range of forward flexion and abduction motion, pain relief, muscle strength, and functionality after RTSA. However, there was no difference in the clinical outcomes of RTSA between patients with cuff tear disease and those with other arthritic diseases.

\section{CONFLICT OF INTEREST}

No potential conflict of interest relevant to this article was reported.

\section{REFERENCES}

1. Hyun YS, Huri G, Garbis NG, McFarland EG. Uncommon indications for reverse total shoulder arthroplasty. Clin Orthop Surg. 2013;5(4):243-55.

2. Al-Hadithy N, Domos P, Sewell MD, Pandit R. Reverse shoulder arthroplasty in 41 patients with cuff tear arthropathy with a mean follow-up period of 5 years. J Shoulder Elbow Surg. 2014;23(11):1662-8.

3. Favard L, Levigne C, Nerot C, Gerber C, De Wilde L, Mole D. Reverse prostheses in arthropathies with cuff tear: are survivorship and function maintained over time? Clin Orthop Relat Res. 2011;469(9):2469-75.

4. Jo SH, Kim JY, Cho NS, Rhee YG. Reverse total shoulder arthroplasty: salvage procedure for failed prior arthroplasty. Clin Orthop Surg. 2017;9(2):200-6.

5. Muh SJ, Streit JJ, Wanner JP, et al. Early follow-up of reverse total shoulder arthroplasty in patients sixty years of age or younger. J Bone Joint Surg Am. 2013;95(20):1877-83.

6. Holcomb JO, Hebert DJ, Mighell MA, et al. Reverse shoulder arthroplasty in patients with rheumatoid arthritis. J Shoulder Elbow Surg. 2010;19(7):1076-84.

7. Boileau P, Watkinson D, Hatzidakis AM, Hovorka I. Neer Award 2005: The Grammont reverse shoulder prosthesis: results in cuff tear arthritis, fracture sequelae, and revision arthroplasty. J Shoulder Elbow Surg. 2006;15(5):527-40. 
Kim et al. Reverse Total Shoulder Arthroplasty According to Primary Diagnosis

Clinics in Orthopedic Surgery • Vol. 12, No. 4, $2020 \bullet$ www.ecios.org

8. Wall B, Nove-Josserand L, O'Connor DP, Edwards TB, Walch G. Reverse total shoulder arthroplasty: a review of results according to etiology. J Bone Joint Surg Am. 2007;89(7):147685.

9. Kilic M, Berth A, Blatter G, et al. Anatomic and reverse shoulder prostheses in fracture sequelae of the humeral head. Acta Orthop Traumatol Turc. 2010;44(6):417-25.

10. Constant CR, Murley AH. A clinical method of functional assessment of the shoulder. Clin Orthop Relat Res. 1987;(214):160-4.

11. Ellman H, Hanker G, Bayer M. Repair of the rotator cuff: end-result study of factors influencing reconstruction. J Bone Joint Surg Am. 1986;68(8):1136-44.

12. Sirveaux F, Favard L, Oudet D, Huquet D, Walch G, Mole D. Grammont inverted total shoulder arthroplasty in the treatment of glenohumeral osteoarthritis with massive rupture of the cuff: results of a multicentre study of 80 shoulders. J Bone Joint Surg Br. 2004;86(3):388-95.

13. Rockwood CA Jr, Williams GR Jr, Burkhead WZ Jr. Debridement of degenerative, irreparable lesions of the rotator cuff. J Bone Joint Surg Am. 1995;77(6):857-66.

14. Fenlin JM Jr, Chase JM, Rushton SA, Frieman BG. Tuberoplasty: creation of an acromiohumeral articulation-a treatment option for massive, irreparable rotator cuff tears. J Shoulder Elbow Surg. 2002;11(2):136-42.

15. Burkhart SS, Nottage WM, Ogilvie-Harris DJ, Kohn HS, Pachelli A. Partial repair of irreparable rotator cuff tears. Arthroscopy. 1994;10(4):363-70.

16. Gerber C, Rahm SA, Catanzaro S, Farshad M, Moor BK. Latissimus dorsi tendon transfer for treatment of irreparable posterosuperior rotator cuff tears: long-term results at a minimum follow-up of ten years. J Bone Joint Surg Am. 2013;95(21):1920-6.

17. Zuckerman JD, Scott AJ, Gallagher MA. Hemiarthroplasty for cuff tear arthropathy. J Shoulder Elbow Surg. 2000;9(3):169-72.

18. Gerber C, Pennington SD, Nyffeler RW. Reverse total shoulder arthroplasty. J Am Acad Orthop Surg. 2009;17(5):28495.

19. Boileau P, Watkinson DJ, Hatzidakis AM, Balg F. Grammont reverse prosthesis: design, rationale, and biomechanics. J Shoulder Elbow Surg. 2005;14(1 Suppl S):147S-61S.

20. Mizuno N, Denard PJ, Raiss P, Walch G. Reverse total shoulder arthroplasty for primary glenohumeral osteoarthritis in patients with a biconcave glenoid. J Bone Joint Surg Am. 2013;95(14):1297-304.

21. Rittmeister M, Kerschbaumer F. Grammont reverse total shoulder arthroplasty in patients with rheumatoid arthritis and nonreconstructible rotator cuff lesions. J Shoulder Elbow Surg. 2001;10(1):17-22.

22. Padegimas EM, Nicholson TA, Silva S, et al. Outcomes of shoulder arthroplasty performed for postinfectious arthritis. Clin Orthop Surg. 2018;10(3):344-51.

23. Patel DN, Young B, Onyekwelu I, Zuckerman JD, Kwon YW. Reverse total shoulder arthroplasty for failed shoulder arthroplasty. J Shoulder Elbow Surg. 2012;21(11):1478-83.

24. Statz JM, Schoch BS, Sanchez-Sotelo J, Sperling JW, Cofield RH. Shoulder arthroplasty for locked anterior shoulder dislocation: a role for the reversed design. Int Orthop. 2017;41(6):1227-34.

25. Lazerges C, Dagneaux L, Degeorge B, Tardy N, Coulet B, Chammas M. Composite reverse shoulder arthroplasty can provide good function and quality of life in cases of malignant tumour of the proximal humerus. Int Orthop. 2017;41(12):2619-25.

26. Frankle M, Siegal S, Pupello D, Saleem A, Mighell M, Vasey M. The reverse shoulder prosthesis for glenohumeral arthritis associated with severe rotator cuff deficiency: a minimum two-year follow-up study of sixty patients. J Bone Joint Surg Am. 2005;87(8):1697-705.

27. Willis M, Min W, Brooks JP, et al. Proximal humeral malunion treated with reverse shoulder arthroplasty. J Shoulder Elbow Surg. 2012;21(4):507-13.

28. Martinez AA, Bejarano C, Carbonel I, Iglesias D, GilAlbarova J, Herrera A. The treatment of proximal humerus nonunions in older patients with reverse shoulder arthroplasty. Injury. 2012;43 Suppl 2:S3-6.

29. Cuff D, Pupello D, Virani N, Levy J, Frankle M. Reverse shoulder arthroplasty for the treatment of rotator cuff deficiency. J Bone Joint Surg Am. 2008;90(6):1244-51.

30. Cuff DJ, Virani NA, Levy J, et al. The treatment of deep shoulder infection and glenohumeral instability with debridement, reverse shoulder arthroplasty and postoperative antibiotics. J Bone Joint Surg Br. 2008;90(3):336-42.

31. Levigne C, Garret J, Boileau P, Alami G, Favard L, Walch G. Scapular notching in reverse shoulder arthroplasty: is it important to avoid it and how? Clin Orthop Relat Res. 2011;469(9):2512-20. 